This document is the accepted manuscript version of the following article:

Leclaire, N. A., Li, M., Véron, A. C., Neels, A., Heier, J., Reimers, J. R., \& Nüesch, F. A. (2018). Cyanine platelet single crystals: growth, crystal structure and optical spectra. Physical Chemistry Chemical Physics. https://doi.org/10.1039/ C8CP06034G

\title{
Cyanine Platelet Single Crystals: growth, crystal structure and optical spectra
}

Nicolas A. Leclaire ${ }^{1,2}$, Musen $\mathrm{Li}^{3}$, Anna C. Véron ${ }^{1}$, Antonia Neels ${ }^{4}$, Jakob Heier ${ }^{1}$, Jeffrey R. Reimers $^{3,5^{*}}$, Frank. A. Nüesch ${ }^{1,2^{*}}$

${ }^{1}$ Empa, Laboratory for Functional Polymers, Swiss Federal Laboratories for Materials Science and Technology, Überlandstrasse 129, 8600 Dübendorf, Switzerland

${ }^{2}$ Institut des Matériaux, École Polytechnique Fédérale de Lausanne, Station 12, 1015 Lausanne, Switzerland

${ }^{3}$ International Center for Quantum and Molecular Structures, College of Science, Shanghai University, Shanghai, 200444, China

${ }^{4}$ Empa, Center for X-Ray Analytics, Swiss Federal Laboratories for Materials Science and Technology, Überlandstrasse 129, 8600 Dübendorf, Switzerland

${ }^{5}$ School of Mathematical and Physical Sciences, University of Technology Sydney, Sydney, 2007, Australia

\begin{abstract}
Crystalline organic semiconducting materials are much in demand for multiple electronic and optoelectronic device applications. Here, solution grown ultrathin rhombic crystals of a trimethine carbocyanine anionic dye are used to establish relationships between structural and optical properties. The dye crystallized in the monoclinic space group $\mathrm{P} 2_{1} / \mathrm{c}$ featuring alternating layers of molecules in two different herringbone type patters, with perchlorate counterions located mostly within one of the two layers. Micro transmittance spectroscopy revealed a broadened spectrum compared to those obtained in solution and in an amorphous thin film. Using polarized light, transmission spectroscopy revealed strong low-energy and weak high-energy bands polarized along the crystallographic b- and c-axis, respectively. Using the extended dipole approximation, significant exciton couplings are predicted between neighboring molecules in the crystal, of the order of the intrinsic monomer reorganization energies associated with nuclear relaxation after excitation, depicting a complex spectral scenario. The exciton coupling pattern explains the relative energies of the b- and c- polarized components but the observed intensities are opposite to expectations based on chromophore alignment within the crystal.
\end{abstract}




\section{Introduction}

The condensed-phase anisotropic structures of planar organic $\pi$-conjugated molecules and their salts or coordination compounds are determined from a combination of the involved chemical forces and the native inter-chromophore van der Waals forces. The characteristics of semiconducting solids are strongly related to the resulting intermolecular overlap of the frontier orbitals. In these solids, charge transport, ${ }^{[1,2]}$ exciton diffusion, ${ }^{[3,4]}$ absorption, ${ }^{[5]}$ charge-transfer ${ }^{[6]}$ and energy-level alignment ${ }^{[7]}$ are typical orientation-dependent attributes. These properties are essential to the performances of optoelectronic devices based on organic semiconductors (OSCs) such as organic field-effect transistors (OFETs) ${ }^{[8-10]}$ organic photovoltaic (OPV) devices ${ }^{[1,12]}$ and organic light-emitting diodes (OLEDs) ${ }^{[13]}$.

An approach to optimize OSC devices' performances would therefore require control over the molecular orientation in the active layers together with smart device design. A strategy to control the orientation of molecules within active layers is to fabricate crystalline films, which provide long range order and defined molecular orientation. Due to technological limitations in the fabrication processes of organic crystalline films from semiconducting small molecules, the field of organic optoelectronics has for a long time mainly focused on the use of amorphous layers. ${ }^{[14]}$ To be relevant in most device applications, crystalline active layers should be (i) thin (typically smaller than $\sim 100 \mathrm{~nm}$ due to the short diffusion length of charges and excitons), (ii) free from defects (structural and chemical) and (iii) free from grain boundaries which are detrimental to transport properties. The choice of materials and fabrication methods has therefore been mostly aligned to meet these requirements. There are, however, only a few systematic investigations correlating the molecular packing of thin crystalline organic semiconductors to their corresponding optical and electronic properties. ${ }^{[15-}$

${ }^{17]}$ An exception is the field of OFETs where single crystal and device fabrication methods ${ }^{[18]}$ have now been optimized with performances competing with their inorganic amorphous 
counterparts. ${ }^{[19]}$ As to luminescent properties, however, strong solid state quenching has always been detrimental both in single crystals and amorphous films but its origin is still not well understood. ${ }^{[20-22]}$ In this context, it is interesting to explore the structure-properties relationship of thin single crystals of OSC materials.

Cyanine dyes are a class of organic semiconductors well known for their strong absorption and emission properties in the visible and near-infrared range. ${ }^{[23,24]}$ In the past cyanines have been mainly used as photosensitizers for silver halide photography, ${ }^{[25]}$ in recording media technologies ${ }^{[26]}$ or as fluorescent markers for biological applications. ${ }^{[27]}$ They are now being investigated in the form of amorphous thin films as active layers in photovoltaic solar cells, photodetectors and light emitting electro-chemical cells. ${ }^{[28-31]}$ Due to the very intense first optical absorption transition in cyanine chromophores, exciton coupling may dominate the optical properties of the solid. ${ }^{[32]}$ While this phenomenon has been extensively studied in Jand $\mathrm{H}$-aggregates, ${ }^{[33,34]}$ spectroscopic investigations of bulk single crystals have been rather limited so far ${ }^{[35,36]}$ and constricted to reflectometry.

This work presents the crystal structure and the anisotropic optical properties of a thin crystal of a cyanine dye 1-ethyl-2-[3-(1-ethyl-1,3-dihydro-3,3-dimethyl-2H-indol-2-ylidene)-1propen-1-yl]-3,3-dimethyl-3H-indolium perchlorate $(\mathbf{C y C})$

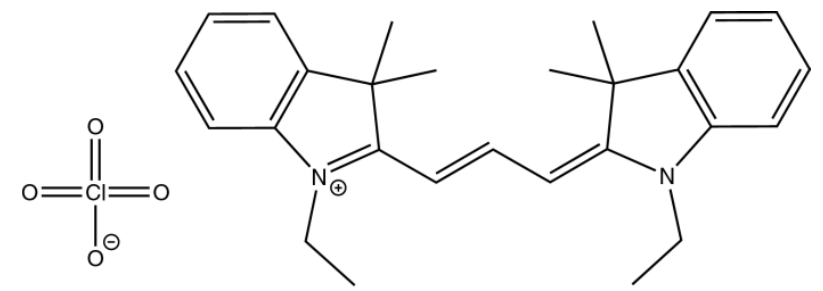

CyC

measured in transmittance. The crystal was grown by solvent-vapor annealing of a dewetted film of the dye solution directly on a solid substrate. ${ }^{[37]}$ The structure of the thin crystal was 
elucidated by X-ray crystal structure analysis. The orientation-dependent optical properties were evaluated by optical spectroscopy on individual crystals. Basic properties of the material are considered using rudimentary density-functional theory (DFT) calculations coupled with some simple estimates using analytical expressions.

\section{Materials and Methods}

\section{CyC Single Crystals Growth}

The $\mathbf{C y C}$ dye used was synthesized in our laboratory. ${ }^{[23]}$ Platelet crystals were fabricated from a saturated solution of the dye $(2.47 \mathrm{mM})$ in isopropyl alcohol solution (anhydrous, 99.5\%, Sigma-Aldrich). First, $\mathbf{C y C}$ droplets were obtained by spin casting the saturated solution on glass substrates. $0.3 \mathrm{~mL}$ of dye solution was first filtered and distributed on the substrates, followed by a slow rotation at $200 \mathrm{rpm}$ for $3 \mathrm{~s}$. The rotational speed was then increased to $1000 \mathrm{rpm}$ at a rate of $3000 \mathrm{rpm} \mathrm{s}^{-1}$ and the samples were spun for $60 \mathrm{~s}$. Subsequently, droplet films were annealed in chlorobenzene (Sigma-Aldrich) vapor overnight in a closed chamber at room temperature.

\section{X-Ray Diffraction}

The small dimensions of the as-deposited platelet crystals on substrates made it impossible to directly derive the 3D single crystal structure. Therefore, single crystal X-ray analysis was performed on large single crystals grown using the vapor diffusion method. A concentrated solution of the dye in chlorobenzene $(44.3 \mathrm{mM})$ was filtered and exposed to isopropyl alcohol vapor in a closed vial. Rhomb-shaped single crystals of few millimeters in size grew after about 4 weeks.

A red block-like shaped crystal of $\mathbf{C y C}$ was mounted on a Stoe Mark II-Imaging Plate Diffractometer System (Stoe \& Cie, 2015) equipped with a graphite-monochromator. Data collection was performed at $-100^{\circ} \mathrm{C}$ using Mo-K $\alpha$ radiation $(\lambda=0.71073 \AA) .180$ exposures 
( 2 min per exposure) were obtained at an image plate distance of $135 \mathrm{~mm}, \varphi=0^{\circ}$ and $0<\omega<$ $180^{\circ}$ with the crystal oscillating through $1^{\circ}$ in $\omega$. The resolution was $D_{\min }-D_{\max } 24.00-0.84$ $\AA$.

The structure was solved by direct methods using the program SHELXS ${ }^{[38]}$ and refined by full matrix least squares on $\mathrm{F}^{[39]}$ with SHELXL ${ }^{[38]}$. The hydrogen atoms were included in calculated positions and treated as riding atoms using SHELXL-97 default parameters. All non-hydrogen atoms were refined anisotropically. CCDC 1863079 contains the supplementary crystallographic data for $\mathrm{CyC}$. These data can be obtained free of charge from The Cambridge Crystallographic Data Centre via www.ccdc.cam.ac.uk/data_request/cif. The drawings were carried out with PLATON. ${ }^{[40]}$

$\mathrm{X}$-ray diffraction (XRD) patterns of platelet samples were obtained from samples containing a large number of platelet crystals as a $\theta-2 \theta$ scan in reflection mode on a PANalytical X'Pert PRO instrument equipped with a germanium monochromator selecting $\mathrm{Cu}-\mathrm{K} \alpha 1$ radiation, $\lambda$ $=1.5406 \AA$. The scattered intensities were recorded using a position-sensitive 1D detector (PANalytical X'Celerator). The detected count rate stayed in the linearity range of the detector. Diffraction patterns were recorded between 3 and $40^{\circ}(2 \theta)$.

\section{Spectroscopic analysis of single crystals}

The transmission spectra of platelet crystals were acquired using a self-built microspectrophotometer. For the absorption measurements, a halogen light source was focused on the crystal sample using a condenser lens and the transmitted light was collected through an Olympus IX-81 microscope connected to an Andor Shamrock 301i spectrometer equipped with an iDUS DV420A camera. When needed, the light was polarized using a polarizing filter placed between the light source and the condenser lens (ESI Figure S1). 
For photoluminescence measurement a green laser $(529 \mathrm{~nm})$ was coupled to a single mode optical fiber focused on the crystal sample. For collection of emitted light a $532 \mathrm{~nm}$ band pass filter was inserted at the entrance slit of the spectrometer.

Fluorescence spectroscopy (Horiba Jobin Yvon Fluorolog) was used to obtain the excitation spectra of a large number of platelet crystals. The measured spectra were corrected to take into account the excitation intensity.

\section{DFT calculations}

The electronic and geometrical structures of the observed crystal and an isolated $\mathrm{CyC}$ cation in solution are modelled using DFT. For the crystal, the VASP program ${ }^{[41,42]}$ is used, with the core electrons treated using the PAW procedure, ${ }^{[43]}$ correlation and exchange using the PBE density functional, ${ }^{[44]}$ the van der Waals attraction treated using D3(BJ) ${ }^{[45]}$ and the basis set determined using the PREC=HIGH flag to set the energy cutoff. The unit cell, containing 8 translationally invariant anions and cations, is orientated to coincide with the spectroscopically observed polarization directions. The Brillouin zone is modelled using, a $2 \times 2 \times 2 \mathrm{~K}-\mathrm{POINT}$ grid. Geometry optimization is performed starting at a symmetry-broken structure, terminating when the maximum force on any atom falls below $0.01 \mathrm{eV} \AA^{-1}$.

For the isolated CyC cation in solution, time-dependent DFT (TDDFT) calculations were performed by Gaussian16 $6^{[46]}$ using the CAM-B3LYP density functional ${ }^{[47,48]}$ (a rangecorrected hybrid functional that represents the entry point for DFT calculations of spectroscopic properties of conjugated molecules ${ }^{[49,50]}$ ) and the $6-31 \mathrm{G}^{*}$ basis set. ${ }^{[51]}$ Solvation was modelled using the PCM method ${ }^{[52]}$ set to model solvation in chloroform. 


\section{Results and Discussion}

\subsection{Growth of CyC Platelet Crystals}

Platelet crystals of $\mathbf{C y C}$ were grown on glass substrate following the method described by Tisserant et al.. ${ }^{[37]}$ A saturated solution of the dye in isopropyl alcohol was first spun cast on clean glass substrates. Upon spin-casting, the dye film dewets and forms an ensemble of micron-sized amorphous droplets which size depends on the rotational speed during the coating process. This spin-casting step was previously identified as the crystal nucleation step. ${ }^{[37]}$
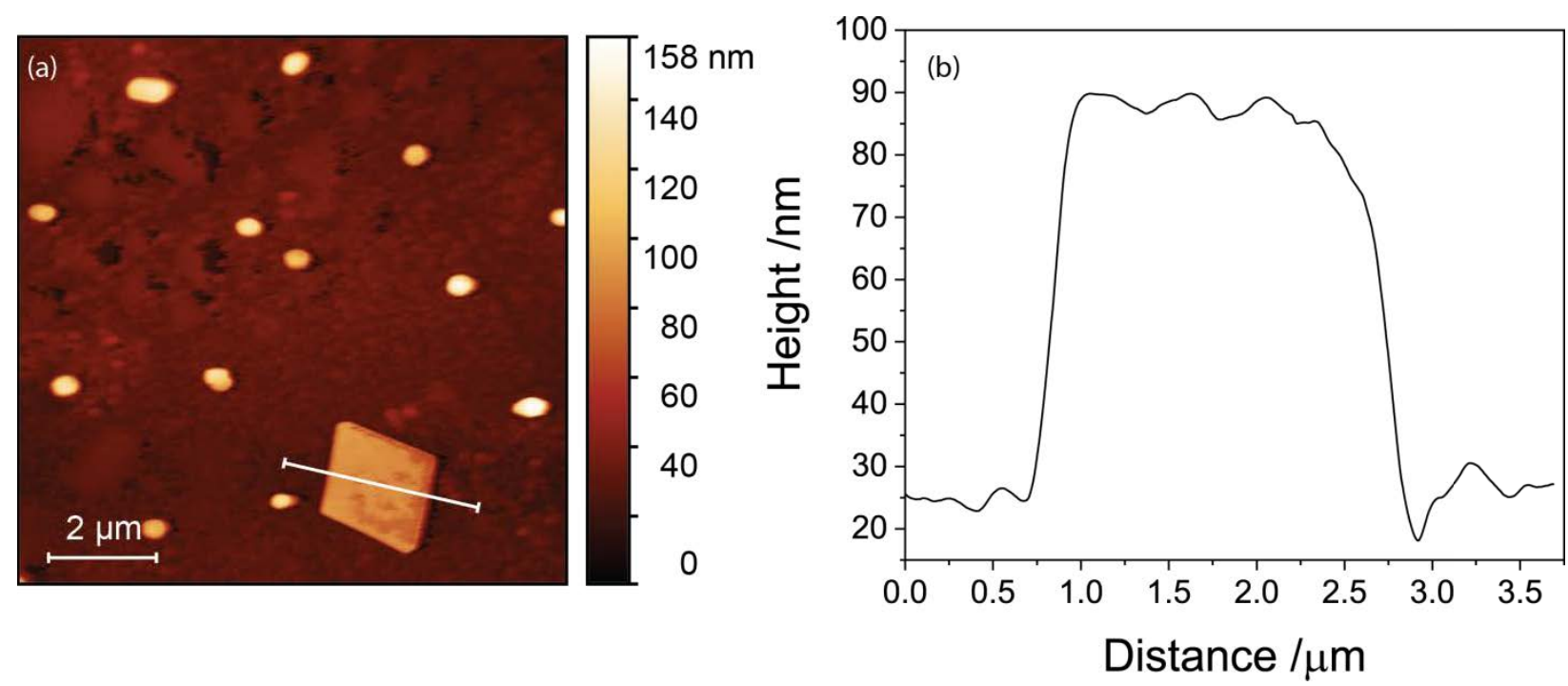

Figure 1: (a) SPM image of a CyC platelet single crystal surrounded by amorphous dye droplets. (b) Profile analysis of the crystal reveals a typical thickness of 50-60 $\mathrm{nm}$.

Platelet crystals grown from droplets have an aspect ratio, which can exceed three orders of magnitude. Figure 1(a) shows a scanning probe microscopy image of a typical $\mathbf{C y C}$ single crystal grown on glass. A few droplets of cyanine dye are also visible surrounding the crystal. The crystal shows a characteristic rhombic shape, with the longest diagonal measuring about $2.7 \mu \mathrm{m}$. Profile analysis (Figure 1(b)) measured at the edge of the crystal in Figure 1(a) shows a crystal thickness of 50-60 $\mathrm{nm}$. The origin of this particular morphology can be 
understood from the crystal growth mechanisms described previously. ${ }^{[37]}$ Thickness increase occurs mostly during the first step, the crystal nucleus being fed by the dye molecules readily available in the parent droplet. In this case, growth is limited by the amount of molecules available in the droplet. In a second step, the crystal continues to grow laterally via diffusion through a thin liquid film of the dye molecules available from the surrounding amorphous droplets towards the crystal side faces. There, the crystal keeps expanding as long as amorphous droplets are available in the surroundings, leading to an important lateral expansion of the crystal.

\subsection{X-Ray Single Crystal Structure Analysis}

Due to their reduced size and fragility, single crystal X-ray analysis could not be directly performed on the platelet crystals. The crystal structure determination was therefore performed on a bulk crystal grown by vapor diffusion. The crystals presented a dark red color and pronounced optical anisotropy under crossed-polarizers. The equivalence between the structures is discussed in the next section.

The crystal structure determination revealed that the dye crystallizes as a monoclinic crystal in the space group $P 2_{1} / c$. The asymmetric unit consists of two dye cations and two perchlorate anions $\mathrm{ClO}_{4}{ }^{-}$resulting in the molecular formula $\left[\left(\mathrm{C}_{27} \mathrm{H}_{33} \mathrm{~N}_{2}\right) \mathrm{ClO}_{4}\right]_{2}$. A set of short CH...O interactions with C...O distances in the range of 3.03(1) and 3.53(1) $\AA$ are found between the dye cations and the perchlorate anions. The two dye cations of the asymmetric unit lay in planes forming $83.9^{\circ}$ with respect to each other. The packing of the crystal structure viewed normal to the a-b plane is shown in Figure 2(a) revealing a layered arrangement in which the dye cations of one type assemble in a structure called "layer 1" and the other in "layer 2". Layer 1 is itself a bilayer made from molecules linked to each other by a 2-fold screw axis; the component layers are called layer 1a and layer $1 \mathrm{~b}$. Inside layers 1 and 
2, different herringbone type arrangements of the dye cations are found. In layer 2, dye cations arrange in a more closely packed herringbone fashion (Figure 2 (c)), with a stacking angle of $74.4^{\circ}$ compared to $84.5^{\circ}$ in layer 1 .

(a)

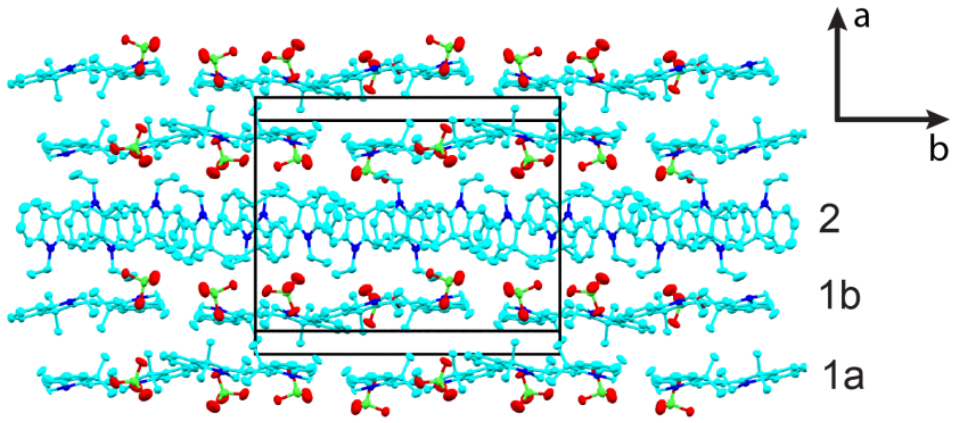

(b)

(c)
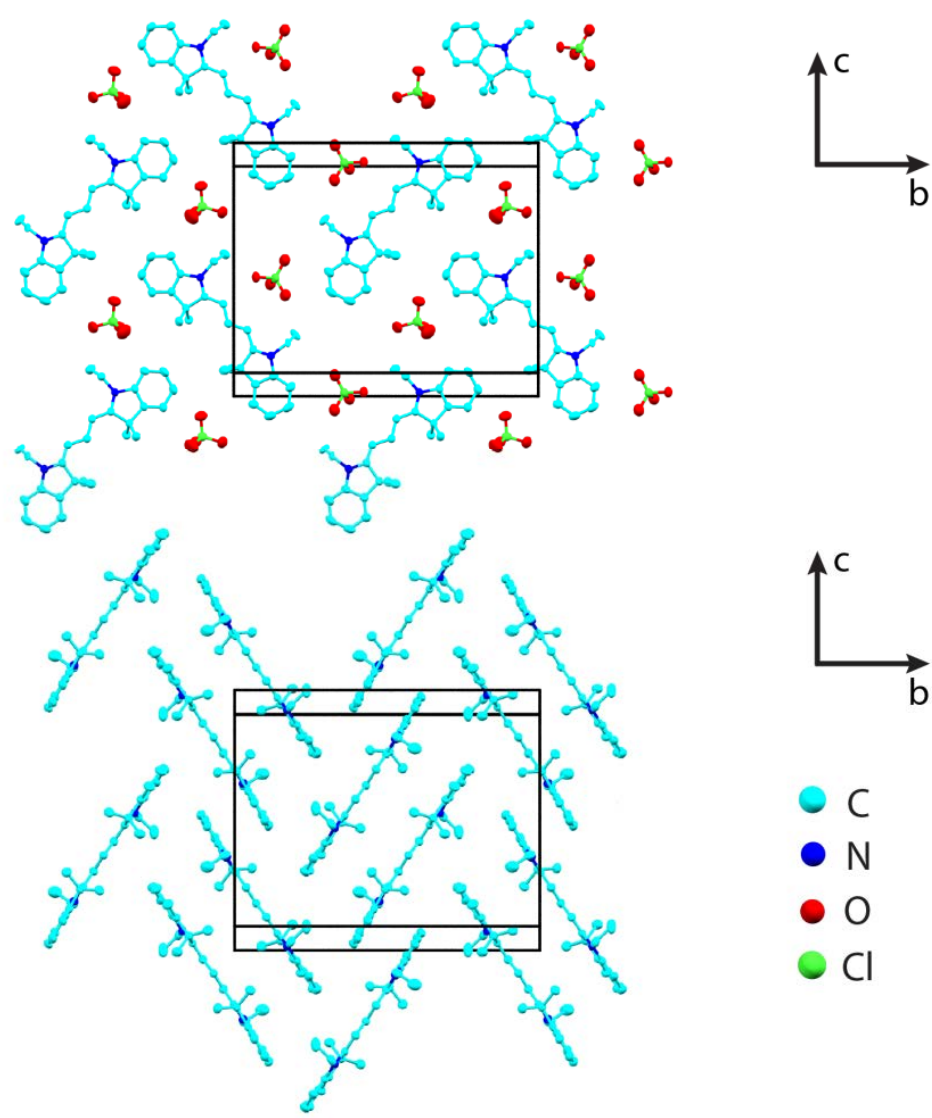

$\mathrm{C}$

- $\mathrm{N}$

- $\mathrm{O}$

$\mathrm{Cl}$

Figure 2: Details of the $\mathbf{C y C}$ crystal packing: (a) Viewed normal to the ab plane, revealing stacked layers 1

(with components 1a and 1b) and 2; (b) Layer 1a viewed normal to the bc plane (layer $1 \mathrm{~b}$ is related by a 2-fold skew axis); and (c) Layer 2 viewed normal to the bc plane. Within each layer, the dye cations pack in different 
herringbone arrangements. Hydrogen atoms are omitted for clarity. The a axis lies normal to $\mathrm{b}$ and at $95.8^{\circ}$ to $\mathrm{c}$. The bold black lines represents the unit cell of the crystal.

The shortest distance between organic molecular planes is $4.162 \AA$. While this is quite close to the van der Waals distance beween $\mathrm{sp}^{3}$ carbons, it is much longer than the value of $\sim 3.3 \AA$ found in tightly packed $\mathrm{sp}^{2}$ structures, suggesting that exciton couplings will be large but not extreme. The distances between the central carbon atoms of the polymethine chain of parallel neighbors are $5.551 \AA$ and $11.422 \AA$, respectively. In layer 1, the molecular planes of the chromophores have a slight tilt angle of $6.31^{\circ}$ with respect to the (100) plane. The chromophores also arrange in a herringbone structure, however the shortest intermolecular distance is considerably larger than in layer 2 with $15.652 \AA$ between the central carbon atoms of the polymethine chains. Interestingly, interlayer distances are rather short in the crystal structure: the shortest center to center distance between cations in layer 1 and 2 is $6.719 \AA$, while it is $7.242 \AA$ in layer 1 . Accordingly, electronic and excitonic couplings are expected to comprise intralayer and interlayer components. In other words, charges and excitons may not only diffuse inside a particular layer but also across layers.

The dye cations in layer 1 have $\mathrm{C}_{2 \mathrm{v}}$ symmetry, making their lowest-energy transition of primary interest herein polarized in the N-N direction. In layer 2, the dye cations have $\mathrm{C}_{2 \mathrm{~h}}$ symmetry, the difference owing to rotation of the orientation of a terminal $\mathrm{CH}_{3}$ group attached to an ethyl sidechain. This effect is expected to have negligible effect on the orientation of the transition polarization, however, and we take the N-N direction as indicative. The N-N vectors are found to orient at $8^{\circ}$ to the face of b-c plane in layer 1 and at $5^{\circ}$ in layer 2 , indicating that almost all absorption will occur within the b-c plane. In layer 1 the polarization within this plane is oriented at $42^{\circ}$ to $\mathrm{c}$, while in layer 2 this angle is $35^{\circ}$. From these angles, the absorption in the c direction is expected to be 1.5 times that in the $b$ direction. 
An important aspect of the crystal is likely to be the arrangement of the perchlorate anions shown in Fig. 2a. These sit mostly within layer 1 and hence provide a very asymmetric electric potential for the dye molecules in layers 1 and 2 . In effect, alternate stacked layers take on formal charges of -1 (layer 1) and +1 (layer 2) per asymmetric unit. Each inter-layer boundary can therefore be thought of as a bulk heterojunction. This is likely to have a profound effect on charge-transfer band spectroscopy, dramatically lowering the energy of appropriately oriented inter-layer charge transfer bands.

\subsection{Out-of-plane orientation of the thin film crystals}

Based on the single crystal analysis, the morphology of the crystal was calculated according to the Bravais-Friedel Donnay-Harker (BFDH) method (ESI Figure S3) ${ }^{[53]}$ Comparing the morphology of the thin platelet crystals in Figure 3(a) and (b), a preferential crystal growth with the $\{\mathrm{h} 00\}$ planes parallel to the substrate is obtained which is also consistent with a layer-by-layer growth mode. ${ }^{[54]}$ The angles formed between the edges of the crystals were measured from optical microscopy images to be $73.5 \pm 1.7^{\circ}$ and $106.1 \pm 1.1^{\circ}$ and agree well with the angles formed by the $\{011\}$ and $\{0-11\}$ faces predicted by the BFDH model of $73.8^{\circ}$ and $106.2^{\circ}$ respectively. The BFDH model also reveals that the c-axis is oriented along the short diagonal axis of the crystal, while the b-axis is oriented along the long diagonal axis. From the morphological point of view, this analysis strongly supports that the bulk single crystal structure is equivalent to the one of the platelet crystals.

$\mathrm{X}$-ray diffraction measurements in $\theta-2 \theta$ mode further confirm the out-of-plane orientation of the crystals. Moreover, the diffraction pattern measured on hundreds of platelet crystals agrees well with the simulated pattern calculated from the single crystal structure analysis assuming the (h00) plane to be parallel to the substrate surface (Figure $3 \mathrm{c}$ ). ${ }^{[55]}$ The measured diffraction pattern shows three main reflections at $2 \theta=5.96^{\circ}, 2 \theta=16.6^{\circ}$ and $2 \theta=22.2^{\circ}$, which 
are attributed to $d_{100}, d_{300}$ and $d_{400}$ respectively, based on the simulated pattern from the single crystal structure. Although the $\mathrm{d}_{200}$ reflex does not correspond to a systematic extinction for this point group, its relative intensity is predicted by the simulation to be low for this preferential orientation compared to the other reflexes of the $\{100\}$ family. ${ }^{[56]}$ Looking more closely at the experimental diffractogram, an additional reflex of small intensity at $\theta=14.21^{\circ}$ is visible, which could be attributed to $\mathrm{d}_{022}$ according to the simulated powder pattern (assuming no preferential orientation). The occurrence of this peak with such low relative intensity may be attributed to the presence of mis-oriented platelet crystals in the sample. Both the geometrical growth model and the crystal diffraction study evidence structure and crystalline orientation of the platelet crystals with the b-c plane being parallel to the substrate surface. This is corroborated by complete extinction of the crystals observed under the optical microscope using crossed polarizers when the polarization is oriented parallel to b or $\mathrm{c}$. Additionally, the conoscopy analysis is consistent with the assignment of crystal orientation and confirms that the crystalline b-axis lies in the substrate plane (ESI Figure S4).
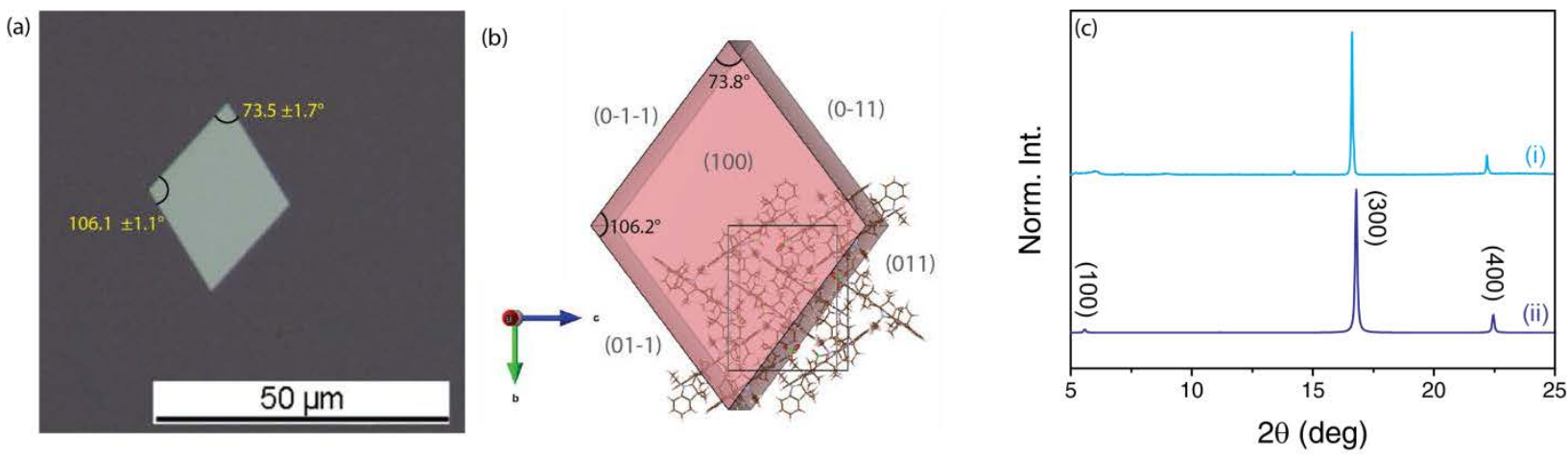

Figure 3:(a) Optical microscopy image (reflection mode) of a platelet crystal. (b) The BFDH morphology predicted from single crystal analysis points towards a preference for the crystal to orient with $\{$ h00\} planes parallel to the substrates. (c) Comparison of $\theta-2 \theta$ diffractogram of platelet crystals with that simulated from single crystal analysis ${ }^{[56]}$ confirms the preferred orientation of the crystals.

\subsection{DFT calculation of the crystal structure}


A DFT optimization of the crystal structure was performed starting with the coordinates obtained previously from X-Ray analysis. Such a single calculation does not incorporate the rotational averaging of the perchlorate ions found in the crystal and hence involve intrinsic symmetry lowering from the observed $P 2_{1} / c$ space group. A total of 8 anions and cations, each with different local environments are included in a translationally equivalent unit cell (see e.g., Fig. 2) and fully optimized. The resulting structure is given in the ESI. Despite starting with a highly asymmetric structure, the material optimized to be very close to $P 2_{1} / c$ symmetry. This result indicates that all anions are essentially equivalent in the structure, consistent with the experimental results. However, it is possible that the observed structure depicts unresolved thermal equilibrium involving chemical forms containing inequivalent ions formed by intermolecular charge transfer. All measured $U$ factors ${ }^{[57]}$ depicting thermal and zero-point motion as well as positional disorder are small (see Figure S5), implying that this is not the case, supported here by the DFT calculations.

\subsection{Optical transmission of platelet crystals}

The low crystal thickness of 50-100 nm enables to directly measure the transmission spectra using a micro-spectrophotometer. As displayed in Figure 4a, the absorption spectrum of the dye in chloroform exhibits two absorption peaks at $18000 \mathrm{~cm}^{-1}$ and $19100 \mathrm{~cm}^{-1}$ corresponding to $0-0$ absorption and $0-1$ vibronic transition of the isolated chromophore, respectively. ${ }^{[58]}$ This spectrum can be fitted to a 3-line Franck-Condon spectral model (ESI Figure S6), with results given in Table 1; the total fitted reorganization energy is $862 \mathrm{~cm}^{-1}$. TDDFT calculations of an isolated dye anion in solution (ESI Table S2) predict that only one transition locates in the broad spectral region of interest, strongly supporting this analysis. 
Table 1. Franck-Condon analysis of the absorption spectrum in chloroform in terms of three modes of frequency $v$ with displacements (in zero-point units) $\delta$, Huang Rhys factors $S=\delta^{2} / 2$, and reorganization energies $\lambda=h v \delta^{2} / 2$

\begin{tabular}{cccc}
\hline$v\left(\mathrm{~cm}^{-1}\right)$ & $\delta$ & $S$ & $\lambda\left(\mathrm{cm}^{-1}\right)$ \\
\hline 1500 & 0.62 & 0.1922 & 288 \\
1050 & 0.86 & 0.3698 & 388 \\
480 & 0.88 & 0.3872 & 186 \\
\hline
\end{tabular}
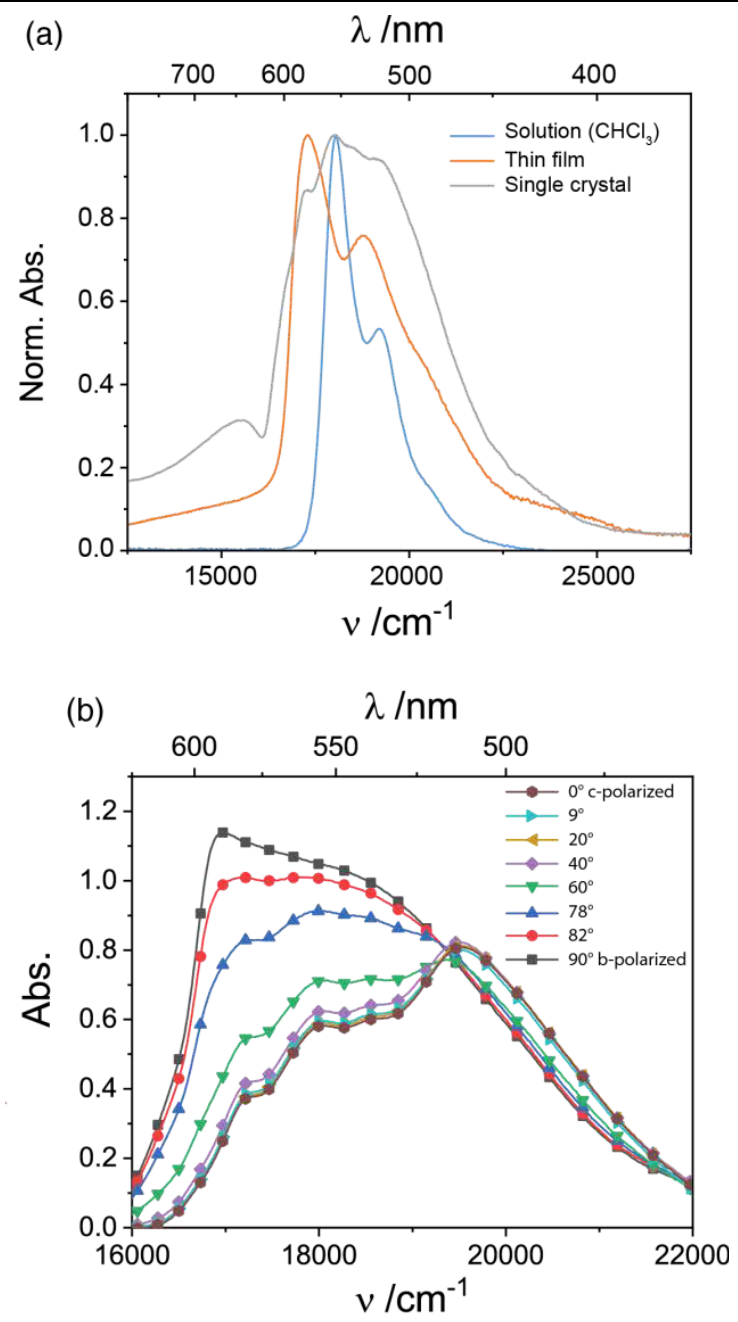

Figure 4: (a) Comparison of absorbance spectra of $\mathbf{C y C}$ in chloroform solution, as an amorphous thin film and in the form of single crystal; (b) Under linearly polarized light, the absorbance of the crystal varies as a function of the crystal orientation; and (c) Relative position of the crystal and light polarization direction (double arrows) at all angles presented in (b).

(c)
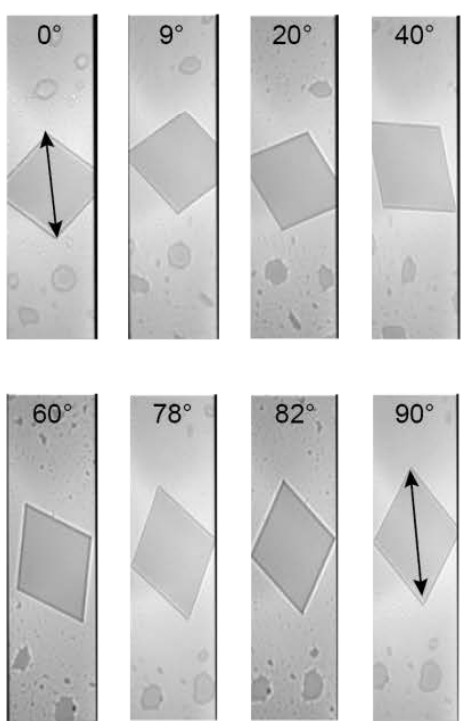
The thin film spectrum in Figure 4a shows two bands, red-shifted compared to the solution spectrum, at $17280 \mathrm{~cm}^{-1}$ and $18790 \mathrm{~cm}^{-1}$ generally attributed to the monomer and dimer absorptions, respectively. The faint shoulder around $20300 \mathrm{~cm}^{-1}$ is attributed to H-aggregates forming in the film. In comparison, the single crystal exhibits a larger absorption bandwidth which can be decomposed into 4 peaks at about $16700 \mathrm{~cm}^{-1}, 17200 \mathrm{~cm}^{-1}, 18000 \mathrm{~cm}^{-1}$ and $19100 \mathrm{~cm}^{-1}$. The peak at $15500 \mathrm{~cm}^{-1}$ did not appear consistently in every crystal and is attributed to thin film interference induced by the interfaces at the crystal surfaces. This artefact is difficult to avoid, however it is reasonable to neglect it in the strongly absorbing region of the spectrum, i.e. $17000-20000 \mathrm{~cm}^{-1}$. Measurement of excitation spectra (ESI Figure S7) confirmed the presence of peaks corresponding to the $19100 \mathrm{~cm}^{-1}$ and $17200 \mathrm{~cm}^{-1}$ bands, with however a larger bandwidth at high energies. This could be explained by the fact that excitation spectra were measured on a large number of small crystals and the resulting spectra are therefore an average of the different crystalline platelet contributions. Indeed, absorbance cannot be simply related to the transmittance signal due to interference and reflectance effects which lead to marked differences in the relative intensity of the bands as well as variations in the peak positions of several $100 \mathrm{~cm}^{-1}$. The apparent similarity of the observed crystal emission spectra to the observed solution absorption spectrum, and the disparity between its shape and that expected for a charge-transfer transition indicates that the lowest-energy components of the excited-state profile are not dominated by charge-transfer effects.

At this stage it is difficult to determine which effects are responsible for the broadening of the crystal absorbance spectrum. Vibronic and excitonic coupling, as well as site- and Starkeffects, can influence the spectral envelope substantially. The attempt to fit the crystal 
spectrum assuming only vibronic contributions, however, did not provide a satisfactory match to the observed spectrum.

A rough estimation of the exciton coupling strength in the layered crystal structure is obtained by evaluating the exciton coupling energy of neighboring pairs in the crystal structure using the extended dipole model ${ }^{[59]}$ (see ESI). The strongest exciton coupling energies found in layer 2 are $263 \mathrm{~cm}^{-1},-219 \mathrm{~cm}^{-1}$ and $127 \mathrm{~cm}^{-1}$. Interlayer coupling energies of $172 \mathrm{~cm}^{-1}$ and $107 \mathrm{~cm}^{-1}$ (between layer 1 and 2) as well as $138 \mathrm{~cm}^{-1}$ and $120 \mathrm{~cm}^{-1}$ (between sub layers 1a and 1b) are also predicted. Given the geometrical arrangement of the transition dipole moments in these individual layers, only one excitonic band is expected for each of the $\mathrm{b}$ and $\mathrm{c}$ polarization directions, with that in the $\mathrm{c}$ direction blue shifted compared to that in the $\mathrm{b}$ direction.

Analysis of the polarization dependence of the absorbance supports this hypothesis. Figure 4(b) shows polarized spectra taken at various polarization angles in the b-c plane with respect to the c-axis. From Figures 4(b) and (c) it is apparent that the intensity of the (stronger) absorption band at $17000 \mathrm{~cm}^{-1}$ is maximum when light is polarized along the b-axis of the crystal while the $19500 \mathrm{~cm}^{-1}$ band vanishes. When polarized along the $\mathrm{c}$-axis, the $17000 \mathrm{~cm}^{-1}$ band vanishes, while the (weaker) $19500 \mathrm{~cm}^{-1}$ becomes maximum. At polarization angles between c- and b-polarization, the intensity of the $17000 \mathrm{~cm}^{-1}$ band decreases to the benefit of the $19500 \mathrm{~cm}^{-1}$ band which increases correspondingly.

The maximum separation between the b- and c- polarized bands would be of order 4 times the exciton coupling strength, of order $1000 \mathrm{~cm}^{-1}$ based on the above predictions of the extended-dipole model. This value is of the order of the intrinsic spectral reorganization energy $\left(862 \mathrm{~cm}^{-1}\right.$ from Table 1$)$, suggesting that a complex interplay between vibrational and excitonic effects determine the spectral lineshape. However, the observed spectral bands are 
much wider than expected based on these features, suggesting that either strong Stark effects dominate the spectrum, charge-transfer bands are evident, and/or the crudely estimated exciton coupling constants from the extended-dipole model are too small by a factor of two.

Besides the bandwidth, another feature of the observed spectra that is not consistent with expectations is the relative intensity distribution. Based on the orientations of the N-N vectors reported earlier, absorption in the c direction is expected to be more intense and blue-shifted compared to absorption in the $\mathrm{b}$ direction. The observed results are in accord with the expected blue-shift of the c-polarized band, but the c:b intensity ratio is ca. 0.8 compared to the anticipated value of 1.5 . The predicted intensity ratio naively depends only on the crystallographic coordinates and is independent of exciton or vibronic coupling effects and so the observed inverted ratio is of significant concern.

It has to be underlined that reflection and interference features complicate the interpretation of the observed transmission spectra. In particular, reflection is important and can reach up to $38 \%$ for strong resonances (ESI Figure S8). This results in an increase and slight broadening of the spectral features of strong absorption bands with respect to weaker ones. When polarized along the b-axis, the main peak at about $19500 \mathrm{~cm}^{-1}$ bears a broad, featureless shoulder extending to about $22000 \mathrm{~cm}^{-1}$. When light is polarized along the c-direction, the spectrum exhibits 3 features of increasing relative intensity at $17250 \mathrm{~cm}^{-1}, 18000 \mathrm{~cm}^{-1}$ and $19500 \mathrm{~cm}^{-1}$. In that orientation, the effect of the reflection seems to be less important, however intensity and peak position variations were also noticed from crystals with differing thicknesses (ESI Figure S9). Unfortunately, the exact contribution of the reflection and interference at different orientations could not be directly measured due to the small size of the crystals. Although a clear polarization dependence with two main axis along b and c reveals the excitonic nature of some of the spectral features, many bands cannot be completely extinguished at $90^{\circ}$ polarization. 


\section{Conclusions}

In this work, crystals of trimethine cyanine dye were grown directly on glass substrates, through solvent vapor annealing of dewetted films of the dye. The crystals showed platelet morphology, with thicknesses in the range of 50-100 $\mathrm{nm}$ and lateral dimensions of tens of

micrometers. The crystalline structure of the platelets was determined by single crystal X-ray analysis and revealed that the chromophores pack in two layered arrangements. In each layer the dye cations arrange in a herringbone fashion. The resulting exciton couplings were estimated using the extended dipole model which was applied to the molecular packing in the crystal structure. Significant intralayer as well as interlayer coupling energies were found. The polarized absorbance spectra of the platelet crystals were measured and confirmed the importance of excitonic contribution to the crystal absorption, while solution spectra indicate that Franck-Condon vibrational effects revealed vibrational couplings of the same order as these exciton couplings, suggesting that a complicated scenario controls band shape. The crystal structure also suggests that different chromophores experience significantly different local electric potentials and fields, while the ionic nature of the crystal and the close intercyanine separations suggest that charge-transfer bands may also contribute. In particular, the concentration of the perchlorate anions near layer 1 suggests the presence of significant lowenergy inter-layer charge transfer bands. Hence a very complex spectral scenario could actually be involved.

Two important aspects of the relationship between structure and spectral properties were naively anticipated: (1) ignoring charge-transfer bands, absorption is expected based on monomer transition-moment alignment to be more intense in the c direction than in $\mathrm{b}$, and, (2), additionally ignoring Stark and charge-transfer effects, the c-polarized band should be blue-shifted compared to the b-polarized one. The first, and most important, correlation was not found, the observed c:b intensity ratio being 0.8 while the anticipated ratio based on 
structure was 1.5. Measuring this property is made quite difficult by the small nature of the microcrystals studied and the associated optical interference and reflection effects, and it is feasible that these are the cause of the discrepancy. However, the involvement of intense charge-transfer bands is another option. The second correlation was observed, with cpolarized intensity being significantly blue-shifted. However, the observed bandwidth is nearly double that expected based on structure and simple estimates of exciton-coupling strengths.

Many questions therefore remain concerning the origin of light absorption in this cyanine dye crystal. In a subsequent work, we examine the issues based on a priori estimates of all quantities involved, including exciton coupling strengths, site and Stark effects, vibronic coupling effects, and charge transfer.

\section{Acknowledgments}

We acknowledge funding from the Swiss National Science Foundation (Grant No. 200021_152909). We thank the Chinese National Natural Science Foundation for support through Grant \#11674212 and the High-End Foreign Expert Program for supporting the collaboration between ICQMS-SHU and Empa. We thank Ole F. Göbel for the conoscopy measurement and Arndt Remhof for his help with X-ray diffraction measurements.

\section{References}

\section{TOC graphic}



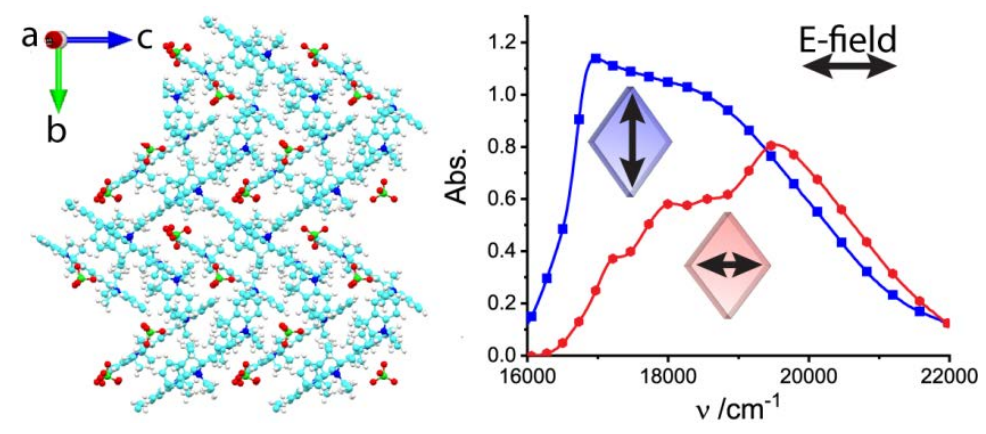

\section{Associated content}

\section{Electronic Supporting Information}

The Electronic Supporting Information (ESI) is available free of charge on the ACS Publications website at DOI:

Experimental details and supporting figures (PDF)

\section{Author information}

corresponding Authors

*J. R. Reimers: E-mail: Jeffrey.Reimers@uts.edu.au

*F.A.Nüesch: E-Mail: frank.nueesch@empa.ch

\section{ORCID}

Frank A. Nüesch : 0000-0003-0145-7611

Anna C. Véron: 0000-0003-3699-7239

Jakob Heier: 0000-0003-2189-3162

Jeffrey R. Reimers: 0000-0001-5157-7422

Musen Li: 0000-0001-9844-8994

\section{Notes}

The authors declare no competing financial interest. 
[1] V. Coropceanu, J. Cornil, D. A. da Silva, Y. Olivier, R. Silbey, J. L. Bredas Chem. Rev. 2007, 107, 926.

[2] V. C. Sundar, J. Zaumseil, V. Podzorov, E. Menard, R. L. Willett, T. Someya, M. E. Gershenson, J. A. Rogers Science 2004, 303, 1644.

[3] G. M. Akselrod, P. B. Deotare, N. J. Thompson, J. Lee, W. A. Tisdale, M. A. Baldo, V. M. Menon, V. Bulović Nat Commun 2014, 5, 3646.

[4] P. Irkhin, I. Biaggio Physical review letters 2011, 107, 017402.

[5] P. Irkhin, A. Ryasnyanskiy, M. Koehler, I. Biaggio Physical Review B 2012, 86, 085143.

[6] Y. H. L. Lin, M. A. Fusella, B. P. Rand Advanced Energy Materials 2018, 8, 1702816.

[7] B. P. Rand, D. Cheyns, K. Vasseur, N. C. Giebink, S. Mothy, Y. Yi, V. Coropceanu, D. Beljonne, J. Cornil, J.-L. Brédas, J. Genoe Advanced Functional Materials 2012, 22, 2987.

[8] A. R. Murphy, J. M. J. Fréchet Chem. Rev. 2007, 107, 1066.

[9] W. H. Lee, J. Park, S. H. Sim, S. Lim, K. S. Kim, B. H. Hong, K. Cho J. Am. Chem. Soc. 2011, 133, 4447.

[10] S. B. Jo, H. H. Kim, H. Lee, B. Kang, S. Lee, M. Sim, M. Kim, W. H. Lee, K. Cho ACS nano 2015, 9, 8206.

[11] C. Videlot, A. El Kassmi, D. Fichou Solar Energy Materials and Solar Cells 2000, 63, 69.

[12] H. C. Han, C. A. Tseng, C. Y. Du, A. Ganguly, C. W. Chong, S. B. Wang, C. F. Lin, S. H. Chang, C. C. Su, J. H. Lee, K. H. Chen, L. C. Chen Journal of materials chemistry 2012, 22, 22899.

[13] D. Yokoyama Journal of materials chemistry 2011, 21, 19187.

[14] S. Ahmad Journal of Polymer Engineering 2014, 34, 279.

[15] G. Lanzani Photophysics of molecular materials: from single molecules to single crystals; John Wiley \& Sons, 2006.

[16] M. Brinkmann, G. Gadret, M. Muccini, C. Taliani, N. Masciocchi, A. Sironi J. Am. Chem. Soc. 2000, 122, 5147.

[17] E. Umbach, K. Glockler, M. Sokolowski Surface Science 1998, 402, 20.

[18] Y. Diao, L. Shaw, Z. Bao, S. C. B. Mannsfeld Energy \& Environmental Science 2014, 7, 2145.

[19] H. Sirringhaus Advanced Materials 2014, 26, 1319.

[20] Y. Q. Zhang, G. Y. Zhong, X. A. Cao Journal of Applied Physics 2010, 108, 083107.

[21] A. P. Green, A. R. Buckley Physical Chemistry Chemical Physics 2015, 17, 1435.

[22] F. A. Castro, H. Benmansour, J.-E. Moser, C. F. O. Graeff, F. Nueesch, R. Hany Physical Chemistry Chemical Physics 2009, 11, 8886.

[23] T. Geiger, H. Benmansour, B. Fan, R. Hany, F. Nueesch Macromolecular Rapid Communications 2008, 29, 651.

[24] A. C. Veron, H. Zhang, A. Linden, F. Nueesch, J. Heier, R. Hany, T. Geiger Organic Letters 2014, 16, 1044.

[25] W. West, P. B. Gilman, T. H. James The Theory of the Photographic Process; Macmillan Pub. Co.: New York, 1977.

[26] K. Y. Law, P. S. Vincett, G. E. Johnson Applied Physics Letters 1981, 39, 718.

[27] L. A. Ernst, R. K. Gupta, R. B. Mujumdar, A. S. Waggoner Cytometry 1989, 10, 3.

[28] M. Bates, R. R. Lunt Sustainable Energy \& Fuels 2017, 1, 955.

[29] H. Zhang, S. Jenatsch, J. De Jonghe, F. Nueesch, R. Steim, A. C. Veron, R. Hany Scientific Reports 2015, 5, 9439.

[30] A. Pertegas, D. Tordera, J. J. Serrano-Perez, E. Orti, H. J. Bolink J. Am. Chem. Soc. 2013, 135, 18008.

[31] S. Jenatsch, L. Wang, N. Leclaire, E. Hack, R. Steim, S. B. Anantharaman, J. Heier, B. Ruhstaller, L. Penninck, F. Nüesch, R. Hany Organic Electronics 2017, 48, 77.

[32] N. J. Hestand, F. C. Spano Accounts of Chemical Research 2017, 50, 341. 
[33] M. Ramanathan, K. Hong, Q. Ji, Y. Yonamine, J. P. Hill, K. Ariga, J. Nanoscience and Nanotechn., 2014, 14, 390.

[34] M. S. Bradley, J. R. Tischler, V. Bulović Advanced Materials 2005, 17, 1881.

[35] A. P. Marchetti, C. D. Salzberg, E. I. P. Walker The Journal of Chemical Physics 1976, 64, 4693.

[36] H. von Berlepsch, S. Möller, L. Dähne J. Phys. Chem. B 2001, 105, 5689.

[37] J.-N. Tisserant, G. Wicht, O. F. Göbel, E. Bocek, G.-L. Bona, T. Geiger, R. Hany, R. Mezzenga, S. Partel, P. Schmid, W. B. Schweizer, J. Heier ACS nano 2013, 7, 5506.

[38] G. M. Sheldrick Acta Crystallogr. Sect. A: Found. Crystallogr. 2008, 64, 112.

[39] A. L. Spek J Appl Cryst, J Appl Crystallogr 2003, 36, 7.

[40] A. L. Spek Acta Crystallographica Section D-Biological Crystallography 2009, 65, 148.

[41] G. Kresse, J. Hafner Physical Review B 1993, 47, 558.

[42] G. Kresse, J. Furthmuller Computational Materials Science 1996, 6, 15.

[43] G. Kresse, D. Joubert Physical Review B 1999, 59, 1758.

[44] J. P. Perdew, K. Burke, M. Ernzerhof Physical review letters 1996, 77, 3865.

[45] L. Goerigk, S. Grimme Physical Chemistry Chemical Physics 2011, 13, 6670.

[46] M. J. Frisch, G. W. Trucks, H. B. Schlegel, G. E. Scuseria, M. A. Robb, J. R. Cheeseman, G. Scalmani, V. Barone, G. A. Petersson, H. Nakatsuji, X. Li, M. Caricato, A. V. Marenich, J. Bloino, B. G. Janesko, R. Gomperts, B. Mennucci, H. P. Hratchian, J. V. Ortiz, A. F. Izmaylov, J. L. Sonnenberg, F. Williams, F. Ding, Lipparini, F. Egidi, J. Goings, B. Peng, A. Petrone, T. Henderson, D. Ranasinghe, V. G. Zakrzewski, J. Gao, N. Rega, G. Zheng, W. Liang, M. Hada, M. Ehara, K. Toyota, R. Fukuda, J. Hasegawa, M. Ishida, T. Nakajima, Y. Honda, O. Kitao, H. Nakai, T. Vreven, K. Throssell, J. A. Montgomery Jr., J. E. Peralta, F. Ogliaro, M. J. Bearpark, J. J. Heyd, E. N. Brothers, K. N. Kudin, V. N. Staroverov, T. A. Keith, R. Kobayashi, J. Normand, K. Raghavachari, A. P. Rendell, J. C. Burant, S. S. Iyengar, J. Tomasi, M. Cossi, J. M. Millam, M. Klene, C. Adamo, R. Cammi, J. W. Ochterski, R. L. Martin, K. Morokuma, O. Farkas, J. B. Foresman, D. J. Fox Gaussian 16 2006, Wallingford.

[47] T. Yanai, D. P. Tew, N. C. Handy Chemical Physics Letters 2004, 393, 51.

[48] R. Kobayashi, R. D. Amos Chemical Physics Letters 2006, 420, 106.

[49] Z. L. Cai, K. Sendt, J. R. Reimers J. Chem. Phys. 2002, 117, 5543.

[50] Z. L. Cai, M. J. Crossley, J. R. Reimers, R. Kobayashi, R. D. Amos Journal of Physical Chemistry B 2006, 110, 15624 .

[51] W. J. Hehre, R. Ditchfield, J. A. Pople J. Chem. Phys. 1972, 56, 2257.

[52] J. Tomasi, B. Mennucci, R. Cammi Chem. Rev. 2005, 105, 2999.

[53] J. D. H. Donnay, D. Harker Am. Mineral 1937, 22, 446.

[54] A. Sassella Crystal Research and Technology 2013, 48, 840.

[55] W. A. Dollase J Appl Cryst, J Appl Crystallogr 1986, 19, 267.

[56] C. F. Macrae, I. J. Bruno, J. A. Chisholm, P. R. Edgington, P. McCabe, E. Pidcock, L. RodriguezMonge, R. Taylor, J. van de Streek, P. A. Wood J Appl Cryst, J Appl Crystallogr 2008, 41, 466.

[57] G. M. Sheldrick Acta Crystallographica Section C-Structural Chemistry 2015, 71, 3.

[58] S. B. Anantharaman, S. Yakunin, C. Peng, M. V. G. Vismara, C. F. O. Graeff, F. A. Nüesch, S. Jenatsch, R. Hany, M. V. Kovalenko, J. Heier J. Phys. Chem. C 2017, 121, 9587.

[59] V. Czikklely, H. D. Forsterling, H. Kuhn Chemical Physics Letters 1970, 6, 207. 